\title{
Produção e qualidade de plantas de gerânio zonal (Pelargonium x hortorum L. H. Bailey) em resposta à aplicação de clormequat, daminozide e paclobutrazol via foliar ${ }^{(1,)}$
}

\author{
SABRINA ARAUJO TINOCO(3); JOSÉ ANTÔNIO SARAIVA GROSSI(3); ARISTÉA ALVES AZEVEDO(4); \\ JOSÉ GERALDO BARBOSA ${ }^{(3)}$ E NERILSON TERRA SANTOS(5)
}

\begin{abstract}
RESUMO
A dinâmica interna do crescimento e desenvolvimento das plantas pode ser modificada mediante a aplicação de certos produtos químicos. O trabalho teve como objetivos observar o efeito da aplicação de doses crescentes de clormequat, daminozide e paclobutrazol em plantas de gerânio zonal (Pelargonium x hortorum L. H. Bailey). Plantas de gerânio das cultivares Grand Prix, Atlantis e Rocky Mountain foram submetidas a três pulverizações foliares nas concentrações de 750, 1000 e $1500 \mathrm{mg}$ i.a./1 de clormequat, 1000, 2000 e $4000 \mathrm{mg}$ i.a./1 de daminozide, 750+2250, 1000+3000 e $1500+4500$ da mistura de clormequat+daminozide e 5, 10 e $20 \mathrm{mg}$ i.a./l de paclobutrazol. A característica dias até a antese não foi afetada por nenhum tratamento. $\mathrm{O}$ daminozide promoveu redução no alongamento das hastes de plantas de gerânio, porém as altas concentrações necessárias para alcançar os resultados desejados podem resultar em custos elevados. As concentrações da mistura de retardantes utilizada proporcionaram maior intensidade de controle do crescimento das plantas quando comparada ao uso do clormequat e daminozide separadamente. Não foram observados sintomas de fitotoxidez, porém o uso da mistura implica manejo mais trabalhoso. Pulverizações foliares com clormequat por três vezes nas concentrações 1000 e $1500 \mathrm{mg}$ i.a./l e com paclobutrazol na concentração de $20 \mathrm{mg}$ i.a./l resultaram em plantas com arquitetura adequada, sendo esse o tratamento recomendado para alcançar os resultados desejados.

Palavras-chave: retardantes de crescimento, aplicação foliar, arquitetura de planta.
\end{abstract}

ABSTRACT

The influence of clormequat, daminozide and paclobutrazol on growth and quality of geranium (Pelargonium x hortorum L. H. Bailey) plants.

Growth retarding activities of chlormequat, daminozide and paclobutrazol were compared on geranium plants (Pelargonium $\mathrm{x}$ hortorum L. H. Bailey). Foliar applications of 750, 1000 e $1500 \mathrm{mg} / \mathrm{l}$ clormequat, 1000, $2000 \mathrm{e} 4000 \mathrm{mg} / 1$ daminozide, $750+2250,1000+3000$ e 1500+4500 tank mix clormequat+daminozide and 5, 10 e $20 \mathrm{mg} / 1$ paclobutrazol were applied for three times at geranium plants cultivars Grand Prix, Atlantis e Rocky Mountain. There were no difference in plant cycle comparing the three retardants. Foliar applications of chlormequat 3 times at 1000 and $1500 \mathrm{mg} / 1$ and paclobutrazol at $20 \mathrm{mg}$ i.a./1 effectively controlled height of all cultivars. Daminozide promotes reductions in stem elongation on geranium plants, but high concentrations needed to achieve desired characteristics may result in excessive costs. Tank mix of chlormequat and daminozide results in higher intensity on control of plant heights, when compared to the use of both growth retardants separately. There were no phytotoxic symptoms observed when tank mix was used, but it requires more labor.

Keywords: growth retardants, foliar applications, plant size.

\section{INTRODUÇÃO}

O setor de floricultura apresenta grande crescimento no cenário agrícola nacional, com a expansão de áreas produtivas e aumento do mercado consumidor. A evolução produtiva do setor representa maior desenvolvimento social e econômico do país, pois emprega cerca de 15 pessoas/ ha (CLARO et al., 2001), um número elevado quando comparado a outras atividades agrícolas. O consumo per capita de flores e plantas ornamentais no Brasil ainda é baixo, quando comparado a outros países (CASTRO, 1998), porém, seu potencial tem mostrado ser o dobro do atual.

As flores de vaso são produtos importantes para o mercado interno, e poucas espécies representam quase a totalidade das plantas comercializadas. A cultura do gerânio tem elevado potencial de consumo, pois, além da rara beleza, as plantas mantêm suas características sob condições domésticas, além disso, as cultivares existentes no mercado podem ser produzidas em várias regiões do país. Em 2003, no Brasil, o cultivo de gerânio representava aproximadamente $1,6 \%$ da área total cultivada para flores em vaso, totalizando 11 ha (IBRAFLOR, 2003).

O cultivo de gerânios zonais em vaso, assim como de outras plantas ornamentais, comumente requer o uso de retardantes de crescimento para obtenção de plantas com arquitetura compatível com o tamanho do vaso, atendendo às exigências do mercado consumidor (BARRETT e HOLCOMB, 1993; TAYAMA e CARVER, 1990). Para os gerânios, o tamanho da planta é representado por um conjunto de aspectos que englobam, além da altura das plantas, a altura da folhagem e o diâmetro da planta. Plantas com características comercialmente desejáveis devem apresentar altura total entre 20 e $25 \mathrm{~cm}$ (DAVIS e CURRY, 1991, HAMZA et al., 1981), altura da folhagem correspondendo a aproximadamente $60 \%$ da altura total da

\footnotetext{
(1) Recebido em 24/05/2010 aceito para publicação em 16/07/2011.

(2) Parte da Dissertação de Mestrado da primeira autora, financiada pelo CNPq.

(3) Departamento de Fitotecnia, UFV, 36570-000, Viçosa, MG.

(4) Departamento de Biologia Vegetal, UFV, 36570-000, Viçosa, MG.

(5) Departamento de Informática, UFV, 36570-000, Viçosa, MG.
} 
planta, entre 12 e $15 \mathrm{~cm}$, e diâmetro entre 1,5 a 2 vezes o maior diâmetro do vaso (CHRISTENSEN e FRIIS, 1987), além de inflorescências com grande diâmetro, produzidas no mais curto período de tempo possível.

A principal função dos retardantes de crescimento é a redução do alongamento das hastes (DAVIS et al., 1988; DAVIS e CURRY, 1991; SACHS e HACKETT, 1972) como resultado da redução nos níveis endógenos de giberelina. A resposta obtida a partir da aplicação dos retardantes de crescimento depende da cultivar, da parte da planta tratada, da idade fisiológica da planta e do seu estádio de desenvolvimento, da quantidade de princípio ativo absorvida e da sua persistência, da forma de aplicação e das condições ambientais (MEJIAS e RUANO, 1990).

Vários fatores de manejo devem ser observados para aplicação de retardantes de crescimento em determinada cultura. A escolha do produto é um fator de grande importância para determinar outros aspectos como o modo de aplicação, a concentração, a frequência de aplicação, a composição do substrato, entre outros. A época de aplicação também é um fator fundamental para alcançar bons resultados, pois os retardantes de crescimento promovem redução na taxa de crescimento das plantas, sendo assim, devem ser aplicados antes de surtos de crescimento. $\mathrm{O}$ custo dos materiais e o tempo de trabalho envolvidos na aplicação também são fatores relevantes a serem considerados (HOLCOMB e WHITE, 1970).

O retardante de crescimento habitualmente recomendado em outros países para a cultura do gerânio é o clormequat (TAYAMA e CARVER, 1990), conhecido pela designação comercial Cycocel. Maior número de aplicações em concentrações mais baixas e a mistura do clormequat com daminozide são algumas medidas adotadas para prevenir o aparecimento dos sintomas de fitotoxidez (BARRETT e HOLCOMB, 1993). A principal vantagem do uso da mistura é proporcionar maior intensidade no controle do crescimento da planta, se comparado à aplicação dos retardantes separadamente (FAUST e LEWIS, 2003), sendo indicada para situações em que o uso individual do clormequat ou daminozide não é suficiente para promover adequado controle da altura (LEWIS et al., 2002). De acordo com o proposto por FAUST e LEWIS (2003) e LEWIS et al. (2002), a proporção entre clormequat e daminozide na mistura deve ser de uma para três partes.

Daminozide é o retardante de crescimento mais comumente utilizado em espécies ornamentais, e uma das principais razões é sua facilidade de uso. B-Nine é o nome do principal produto comercial. $\mathrm{O}$ uso do daminozide na cultura do gerânio é pouco recomendado, pois apresenta pequena atividade nesta espécie (BARRETT e HOLCOMB, 1993; JOSÉ, 2005). Porém, atualmente, no Brasil, o B-Nine é o único retardante de crescimento registrado no Ministério da Agricultura para uso em culturas ornamentais.

O paclobutrazol, conhecido pelo nome comercial de Bonzi ou Pachlobutrazol 100 CE e comercializado sob a forma líquida, é ativo na inibição do crescimento em extensão em um grande número de espécies (DAVIS et al., 1988, RADEMACHER, 2000), incluindo gerânios (BARRETT e HOLCOMB, 1993; NELSON, 2003). O ingrediente ativo paclobutrazol é muito mais eficiente em reduzir o tamanho das plantas que os retardantes de crescimento mais antigos, como o clormequat e o daminozide (OLSEN e ANDERSEN, 1995), além de atuar em maior número de espécies (BARRETT e NELL, 1990).

O uso de paclobutrazol na cultura do gerânio não promove sintomas de fitotoxidez, como ocorre com o clormequat. Porém, sua alta atividade de inibição da síntese de giberelinas representa uma dificuldade no estabelecimento das concentrações adequadas, podendo causar redução excessiva no tamanho das plantas e afetar outras características importantes como a área foliar.

Os objetivos do trabalho foram avaliar as performances dos diferentes tipos de retardantes de crescimento aplicados em concentrações distintas em plantas de gerânio, bem como verificar qual tipo de retardante e respectiva concentração resultam em arquitetura de planta adequada, de acordo com o desejado pelo mercado.

\section{MATERIAL E MÉTODOS}

O experimento foi conduzido no Setor de Floricultura da Universidade Federal de Viçosa, no período de agosto a outubro de 2004, em casa de vegetação.

As plantas de gerânio foram adquiridas na forma de mudas enraizadas, obtidas por propagação vegetativa, com aproximadamente 6 folhas por muda. Foram utilizadas 3 cultivares de gerânio zonal (Pelargonium x hortorum L.H.Bailey): Grand Prix (inflorescências de cor vermelha), Atlantis (inflorescências de cor rosa escura) e Rocky Mountain (inflorescências de cor rosa salmão), provenientes da empresa de produção de mudas Lazzeri Flor Plant ${ }^{\mathbb{B}}$. No momento da chegada das mudas, elas foram individualmente tranşplantadas para vasos plásticos arredondados, com $900 \mathrm{~cm}^{3}$ de volume e maior diâmetro de $13 \mathrm{~cm}$, contendo substrato 3010 fornecido pela empresa Terra do Paraíso ${ }^{\circledR}$.

Após transplantio em casa de vegetação, as plantas foram dispostas em bancadas, espaçadas $25 \times 25 \mathrm{~cm}$ entre si. O manejo da irrigaçãa e fertirrigação foi realizado de acordo com JUNIOR. As plantas receberam apenas água na primeira semana após o transplantio. Na segunda semana, foram aplicados os fertilizantes 15-15-20 (1g/ litro) + MAP (0,05 g/litro) 3 vezes por semana; nos outros dias, as plantas foram irrigadas somente com água, de acordo com a necessidade. Da terceira semana em diante, foram utilizados os fertilizantes solúveis 15-05-30 (1g/ litro) + MAP (0,05 g/litro) uma vez ao dia, e nitrato de cálcio (1g/litro), uma vez por semana. Foram aplicados 60 $\mathrm{ml}$ da solução de fertilizantes por vaso em cada aplicação.

As plantas foram mantidas sem desponta (pinching) em todos os experimentos para evitar respostas interrelacionadas nas dimensões da planta.

Nas aplicações via foliar das soluções contendo os retardantes de crescimento, foram fornecidos $10 \mathrm{ml}$ da solução por planta, volume suficiente para cobrir

*JUNIOR, R.R.C. Notas culturais gerânios - Lazzeri. Comunicação pessoal, 2004 
uniformemente toda a parte aérea. A aplicação foi realizada com o auxílio de um pulverizador manual com capacidade para $500 \mathrm{ml}$. No momento das pulverizações, foi utilizada uma proteção plástica na superfície do vaso para evitar que a solução aplicada atingisse o substrato.

As pulverizações foram realizadas ao final da tarde, após as 16 horas, para permitir que a parte aérea da planta permanecesse em contato com a solução o maior período de tempo possível.

Os princípios ativos foram obtidos dos produtos comerciais CCC 40 Agrometodos (40\% clormequat), B-Nine ( $85 \%$ daminozide) e Pachlobutrazol 100 CE (10 $\%$ paclobutrazol). As soluções contendo os retardantes de crescimento foram preparadas por meio de diluições dos produtos comerciais, utilizando água destilada.

As cultivares Grand Prix, Atlantis e Rocky Mountain foram tratadas via pulverizações foliares por três vezes, sendo a primeira pulverização 15 dias após o transplantio e as pulverizações seguintes realizadas em intervalos de 10 dias, nas seguintes concentrações: $0,750 \mathrm{mg}$ i.a./1, 1000 $\mathrm{mg}$ i.a./1 e 1500mg i.a./l para clormequat ; 0, $1000 \mathrm{mg}$ i.a./1, $2000 \mathrm{mg}$ i.a./1 e $4000 \mathrm{mg}$ i.a./1 para daminozide; 0,5 $\mathrm{mg}$ i.a./1, $10 \mathrm{mg}$ i.a./1 e $20 \mathrm{mg}$ i.a./1 para paclobutrazol; e $0,750+2250 \mathrm{mg}$ i.a/l, $1000+3000 \mathrm{mg}$ i.a/l e $1500+$ $4500 \mathrm{mg}$ i.a/l para a mistura de clormequat + daminozide, respectivamente.

Todas as avaliações tiveram início na décima primeira semana de cultivo. As características avaliadas foram: altura total da planta a partir da superfície do substrato no vaso até o ponto mais alto da planta, altura da folhagem a partir da superfície do substrato no vaso até o ponto mais alto da folhagem, comprimento do pedúnculo, medido da base até sua extremidade, número de inflorescências, número de flores por inflorescência, matéria seca (MS) das inflorescências incluindo os pedúnculos, matéria seca (MS) dos caules, número de folhas, área foliar, matéria seca (MS) das folhas incluindo os pecíolos, antese: número de dias entre o transplantio das mudas até a abertura da primeira flor da primeira inflorescência, diâmetro da planta: média entre duas medidas perpendiculares do diâmetro em vista superior e diâmetro das inflorescências: média entre duas medidas perpendiculares.

Todos os experimentos foram instalados segundo o esquema fatorial no delineamento em blocos ao acaso com três repetições, sendo cada unidade experimental composta por uma planta. Os dados obtidos foram submetidos à análise de variância. As médias dos fatores qualitativos foram comparadas, utilizando o teste de Tukey a 5\% de probabilidade. Para o estudo dos fatores quantitativos, utilizou-se análise de regressão com o teste para falta de ajustamento para escolha do melhor modelo. Os dados foram analisados utilizando o programa SAS (SAS Institute Inc., NC, USA, 2004).

\section{RESULTADOS E DISCUSSÃO}

Considerando os retardantes utilizados, a aplicação da mistura de clormequat + daminozide resultou em média $20 \%$ menor para altura da planta, quando comparada ao daminozide e paclobutrazol aplicados separadamente (Tabela 1). A aplicação de clormequat e paclobutrazol resultou em maior média para número e diâmetro de inflorescência, tendo sido os valores da última característica semelhantes aos das plantas tratadas com daminozide.

Tabela 1. Médias dos retardantes de crescimento $\mathbf{D M Z}=$ Daminozide, Mistura $=$ Clormequat + Daminozide, $\mathbf{C M Q}=$ clormequat, PACLO = Paclobutrazol, aplicados via pulverizações foliares em plantas de gerânio das cultivares Grand Prix, Atlantis e Rocky Mountain, para as características ALTPLA = Altura de planta $(\mathrm{cm}), \mathbf{C O M P P E}=$ Comprimento do pedúnculo $(\mathrm{cm})$, DPLA = Diâmetro de planta $(\mathrm{cm}), \mathbf{A R F O L}=$ Área foliar $\left(\mathrm{cm}^{2}\right), \mathbf{N U M I N F}=$ Número de inflorescências, NFLINF = Número de flores/inflorescência, DINFL = Diâmetro de inflorescência (cm), Viçosa, MG, 2004

Table 1. The effect of DMZ = Daminozide, Mistura $($ Tank Mix $)=$ Clormequat + Daminozide, $\boldsymbol{C M Q}=$ clormequat, $\boldsymbol{P A C L O}$ = Paclobutrazol on specific growth characteristics of geranium (cultivars Grand Prix, Atlantis e Rocky Mountain) plants treated with spray applications. ALTPLA = Final Height (cm), COMPPE = Stem Lenght (cm), DPLA = Plant Diameter $(\mathrm{cm}), \boldsymbol{A R F O L}=$ Leaf area $\left(\mathrm{cm}^{2}\right), \mathbf{N U M I N F}=$ Inflorescences number, NFLINF = Number of flowers/inflorescences, DINFL = Inflorescence diameter (cm), Viçosa, MG, 2004

\begin{tabular}{cccccccc}
\hline $\begin{array}{c}\text { Retardantes de } \\
\text { crescimento }\end{array}$ & $\begin{array}{c}\boldsymbol{A L T P L A} \\
(\mathbf{c m})\end{array}$ & $\begin{array}{c}\text { COMPPE} \\
(\mathbf{c m})\end{array}$ & $\begin{array}{c}\text { DPLA } \\
(\mathbf{c m})\end{array}$ & $\begin{array}{c}\boldsymbol{A R F O L} \\
(\mathbf{c m})^{2}\end{array}$ & NUMINF & $\begin{array}{c}\text { NFLINF } \\
\text { DINFL } \\
(\mathbf{c m})\end{array}$ \\
\hline DMZ & $29,97 \mathrm{a}$ & $15,83 \mathrm{a}$ & $21,45 \mathrm{ab}$ & $636,52 \mathrm{ab}$ & $7,36 \mathrm{~b}$ & $36,75 \mathrm{a}$ & $8,74 \mathrm{a}$ \\
Mistura & $26,04 \mathrm{c}$ & $14,12 \mathrm{~b}$ & $19,98 \mathrm{~b}$ & $553,59 \mathrm{~b}$ & $6,97 \mathrm{~b}$ & $34,04 \mathrm{ab}$ & $7,98 \mathrm{~b}$ \\
CMQ & $27,35 \mathrm{bc}$ & $15,40 \mathrm{a}$ & $22,49 \mathrm{a}$ & $677,29 \mathrm{ab}$ & $9,03 \mathrm{a}$ & $32,04 \mathrm{ab}$ & $8,85 \mathrm{a}$ \\
PACLO & $28,76 \mathrm{ab}$ & $15,39 \mathrm{a}$ & $20,90 \mathrm{ab}$ & $742,17 \mathrm{a}$ & $8,47 \mathrm{a}$ & $30,27 \mathrm{~b}$ & $8,81 \mathrm{a}$ \\
\hline
\end{tabular}

Médias seguidas de pelo menos uma mesma letra na coluna não diferem entre si, a 5\% de probabilidade, pelo teste de Tukey. 
A menor altura da planta (Figura $1 \mathrm{~A}$ ) observada em função de doses crescentes de clormequat está associada à redução tanto da altura da folhagem (Figura $1 \mathrm{~B}$ ), matéria seca do caule (Figura 1 D), quanto ao comprimento do pedúnculo (Figura $1 \mathrm{C}$ ). As concentrações entre $1000 \mathrm{e}$ $1500 \mathrm{mg}$ i.a./l, de acordo com o proposto por BARRETT e HOLCOMB (1993), MEJIAS e RUANO (1990) e NELSON (2003), proporcionaram um conjunto de características que resultaram em plantas com a arquitetura desejada, ou seja, altura de planta entre 20 e $25 \mathrm{~cm}$ e altura de folhagem entre
12 e $15 \mathrm{~cm}$. Pulverizações com clormequat nas diferentes concentrações resultaram em aumento no número de inflorescências (Figura $1 \mathrm{E}$ ), obtendo-se um máximo de 9,7 inflorescências para a concentração de $850 \mathrm{mg}$ i.a./l. De acordo com SEMENIUK e TAYLOR (1970), o aumento no número de inflorescências ocorre devido ao aumento na ramificação lateral, efeito comumente associado à aplicação de clormequat. $\mathrm{O}$ desenvolvimento reprodutivo pode ter sido estimulado em consequência da inibição do crescimento vegetativo.
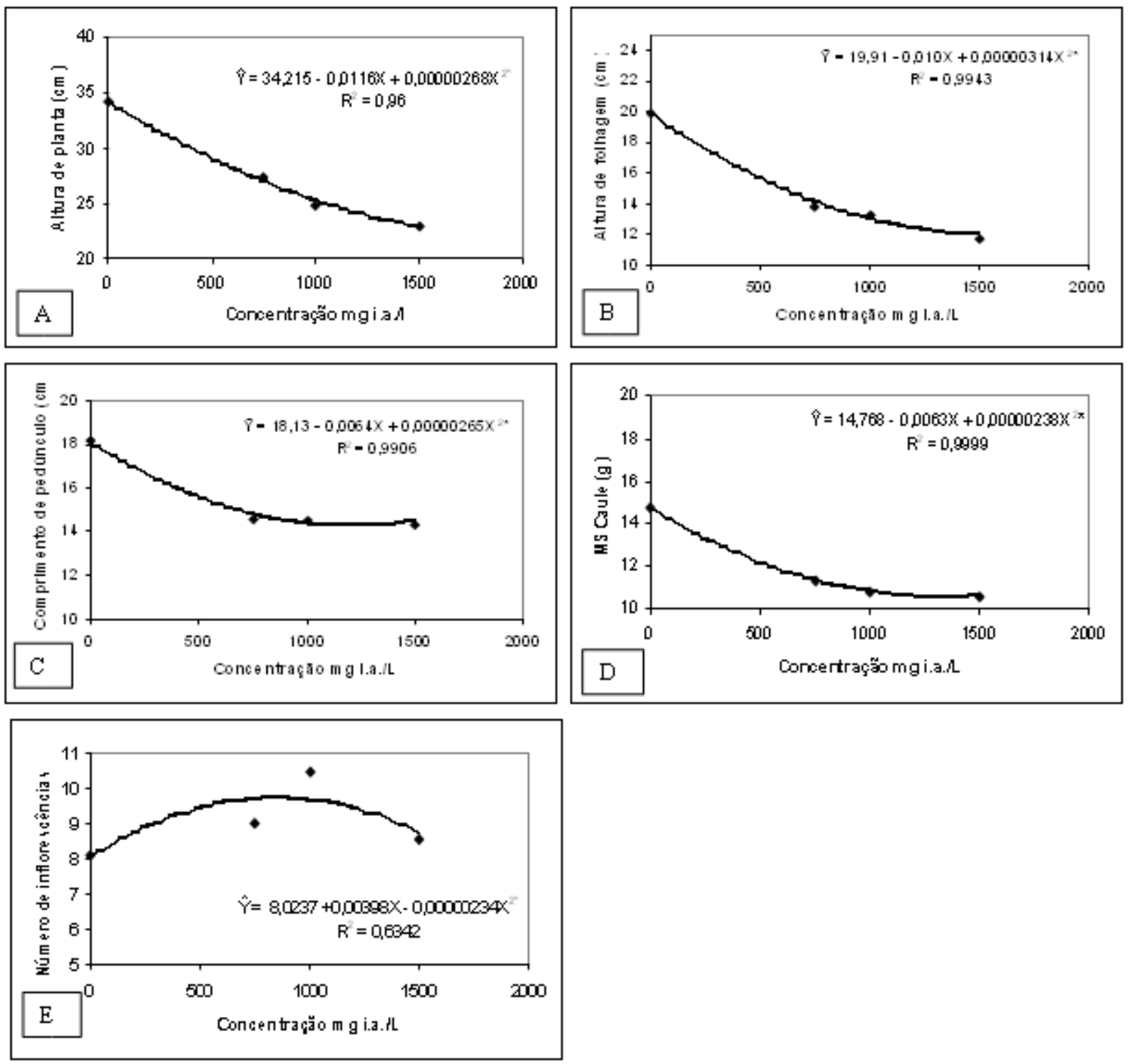

Figura 1. Efeito das concentrações (0, 750, 1000 e $1500 \mathrm{mg}$ i.a./l) de clormequat aplicadas via pulverizações foliares para as características altura de planta (A), altura de folhagem (B), comprimento de pedúnculo (C), matéria seca (MS) do caule (D) e número de inflorescências (E) em plantas de gerânio das cultivares Grand Prix, Atlantis e Rocky Mountain, Viçosa, MG, 2004. significativo a $5 \%$ de probabilidade

Figure 1. Effect of foliar applications of chlormequat (concentrations - 0, 750, 1000 e $1500 \mathrm{mg} / \mathrm{l}$ ) on characteristics plant height (A), foliage height (B), stem lenght (C), shoot dry weight (D) and number of inflorescences (E) on geranium plants cultivars Grand Prix, Ațlantis and Rocky Mountain, Viçosa, MG, 2004. significant at 5\% 
As concentrações de daminozide aplicadas resultaram em menor altura em relação às plantas controle (Figura 2 A), entretanto, o ponto de mínimo da curva, obtido com a concentração de $2756 \mathrm{mg}$ i.a./1, tendeu a apresentar altura de planta de $27,45 \mathrm{~cm}$, superior ao valor máximo de 25 $\mathrm{cm}$ proposto para plantas envasadas. Como o retardante não causou efeito significativo para a característica altura de folhagem, a redução na altura das plantas pode ter sido consequência do efeito significativo do daminozide no comprimento dos pedúnculos das plantas tratadas, que apresentaram valores inferiores aos das plantas controle dentro do intervalo das concentrações aplicadas (Figura 2 B).

A obtenção de plantas com altura superior à desejada neste experimento pode ter ocorrido em função da perda de eficiência do retardante ao longo do ciclo das plantas, conforme observado por TAYAMA e CARVER (1992) em plantas de crisântemo cultivar 'Bright Golden Anne'. Elevadas temperaturas observadas no período de condução do experimento podem ter contribuído para a obtenção de altura final superior à desejada, pois as plantas podem ter retomado rapidamente o crescimento após a aplicação de daminozide, retardante, que não proporciona efeitos em longo prazo de acordo com DICKS e CHARLESEDWARDS (1973).

O diâmetro das plantas submetidas à aplicação de daminozide se reduziu com o aumento das concentrações (Figura $2 \mathrm{C}$ ), observando-se valores adequados para plantas em vaso em todas as concentrações aplicadas. Provavelmente, devido à pequena ação no metabolismo de plantas de gerânio, esse retardante não foi eficiente para reduzir o alongamento da haste principal, evidenciado pela não significância do teste $\mathrm{F}$ para regressão da matéria seca do caule em função da concentração de daminozide, reduzindo, porém, o comprimento dos pedúnculos (Figura 2 B).

A aplicação do daminozide não afetou o número de inflorescências (7,36 inflorescências/planta) (Tabela 2), mas proporcionou efeito significativo no diâmetro destas (Figura $2 \mathrm{D})$, sendo o ponto de mínimo da curva $(8,02 \mathrm{~cm})$ alcançado com a concentração de $2580 \mathrm{mg}$ i.a./l. Concluise, então, que o daminozide pode ter reduzido o tamanho das flores ou o comprimento dos pedicelos, resposta que afeta negativamente o aspecto final das plantas.
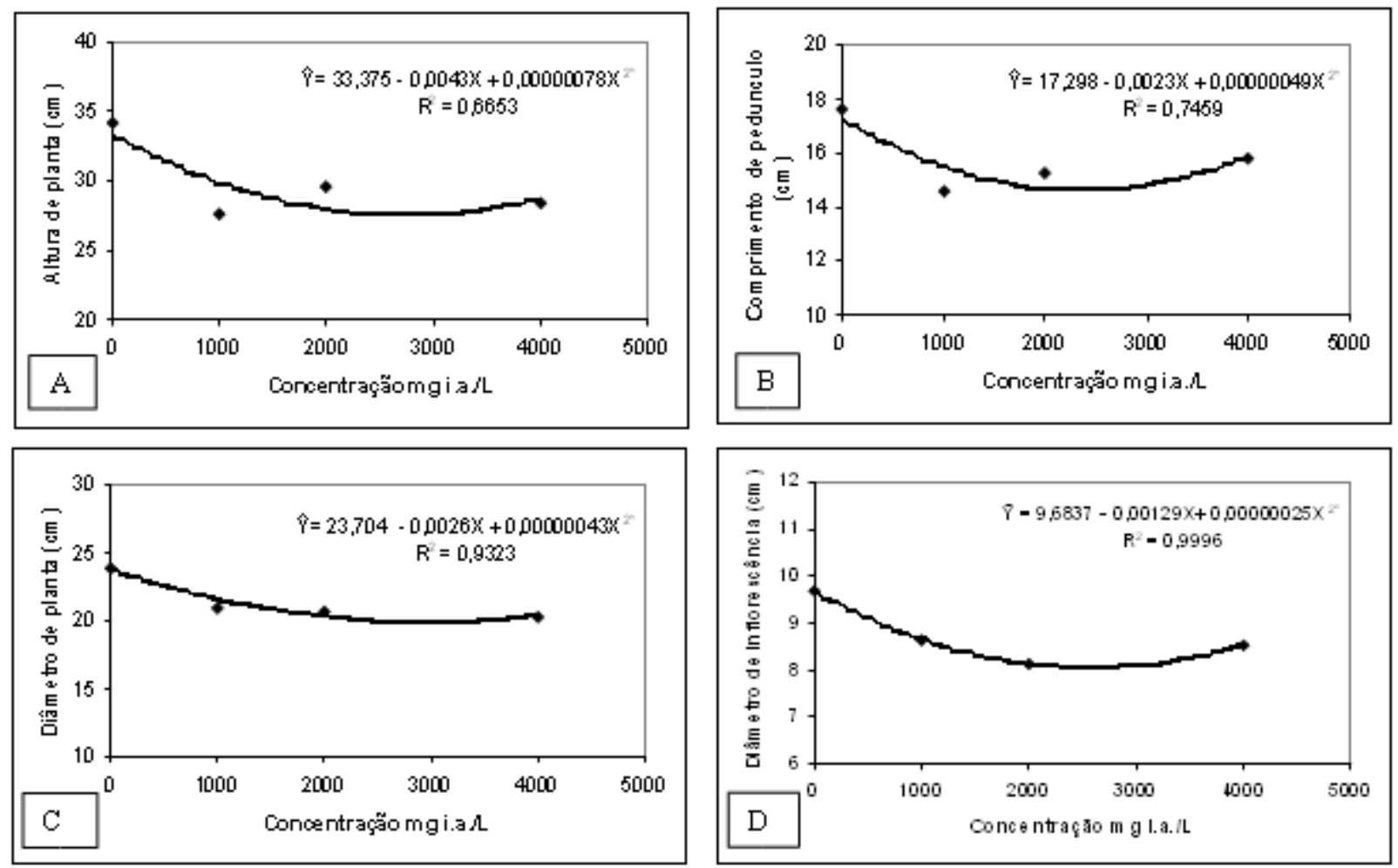

Figura 2. Efeito das concentrações (0, 1000, 2000 e $4000 \mathrm{mg}$ i.a./l) de daminozide aplicadas via pulverizações foliares para as características altura de planta $(\mathrm{A})$, comprimento de pedúnculo (B), diâmetro de planta (C) e diâmetro de inflorescências (D) em plantas de gerânio das cultivares Grand Prix, Atlantis e Rocky Mountain, Viçosa, MG, 2004. significativo a $5 \%$ de probabilidade

Figure 2. Effect of foliar applications of daminozide (concentrations - 0, 1000, 2000 e $4000 \mathrm{mg} / \mathrm{l}$ ) on characteristics plant height (A), stem lenght (B), plant diameter $(C)$ and inflorescences diameter $(D)$ on geranium plants cultivars Grand Prix, Atlantis e Rocky Mountain, Viçosa, MG, 2004. significant at 5\% 
Foram detectadas diferenças significativas entre os níveis de mistura de retardantes clormequat e daminozide para altura da planta, da folhagem e comprimento do pedúnculo (Tabela 2), resultando em plantas com a arquitetura adequada, de acordo com os parâmetros propostos por DAVIS e CURRY (1991) e HAMZA et al. (1981). Porém os valores de diâmetro de planta obtidos foram menores que 19,5, valor mínimo proposto para plantas envasadas, em decorrência da redução da área foliar, muito expressiva quando comparada à ação dos outros retardantes nessa mesma característica.

As concentrações de clormequat utilizadas na mistura foram as mesmas utilizadas nas aplicações individuais, e as concentrações de daminozide foram levemente superiores. Assim, nas plantas tratadas com a combinação de retardantes, a intensidade de controle do crescimento das plantas foi maior, resultando em plantas com aspecto final inferior ao desejado. Esses resultados mostram a ocorrência do efeito aditivo da aplicação do daminozide e clormequat simultaneamente. Ressalta-se a inexistência de sintomas de clorose nas folhas das plantas submetidas às pulverizações com a mistura de clormequat + daminozide, característica observada em plantas tratadas somente com clormequat, mesmo sob concentrações elevadas.

Tabela 2. Médias das características ALTPLA $=$ Altura de planta $(\mathrm{cm}), \mathbf{A L T F O}=$ Altura de folhagem $(\mathrm{cm}), \mathbf{C O M P P E}=$ Comprimento de pedúnculo $(\mathrm{cm})$, DPLA $=$ Diâmetro de planta $(\mathrm{cm}), \mathbf{M S C A}=$ Matéria seca do caule $(\mathrm{g}), \mathbf{A R F O L}=$ Área foliar $\left(\mathrm{cm}^{2}\right)$, MSFOL = Matéria seca das folhas $(\mathrm{g})$ e DINFL = Diâmetro de inflorescências $(\mathrm{cm})$ de plantas de gerânio das cultivares Grand Prix, Atlantis e Rocky Mountain submetidas a concentrações da mistura de clormequat + daminozide (CMQ + DMZ), aplicadas via pulverizações foliares, Viçosa, MG, 2004

Table 2. The effect of Tank Mix = Clormequat + Daminozide (CMQ + DMZ) on specific growth characteristics of geranium (cultivars Grand Prix, Atlantis e Rocky Mountain) plants treated with spray applications. ALTPLA = Final Height (cm), $\boldsymbol{A L T F O}=$ Foliage height (cm), $\boldsymbol{C O M P P E}=$ Stem Lenght (cm), DPLA = Plant Diameter (cm), $\boldsymbol{M S C A}=$ Shoot dry weight $(\mathrm{g}), \boldsymbol{A R F O L}=$ Leaf area $\left(\mathrm{cm}^{2}\right), \mathbf{M S F O L}=$ Leaf dry weight $(\mathrm{g}), \boldsymbol{D I N F L}=$ Inflorescence diameter $(\mathrm{cm})$, Viçosa, $M G, 2004$

\begin{tabular}{|c|c|c|c|c|c|c|c|c|}
\hline $\begin{array}{c}\text { Concentração } \\
\text { mistura } \\
(C M Q+D M Z)\end{array}$ & $\begin{array}{c}A L T P L A \\
(\mathrm{~cm})\end{array}$ & $\begin{array}{c}\text { ALTFO } \\
(\mathrm{cm})\end{array}$ & $\begin{array}{c}\text { COMPPE } \\
(\mathrm{cm})\end{array}$ & $\begin{array}{c}D P L A \\
(\mathrm{~cm})\end{array}$ & $\begin{array}{c}\text { MSCA } \\
(\mathrm{g})\end{array}$ & $\begin{array}{c}A R F Q L \\
\left(\mathrm{~cm}^{2}\right)\end{array}$ & $\begin{array}{c}\text { MSFOL } \\
\quad(g)\end{array}$ & $\begin{array}{c}\text { DINFL } \\
\text { (cm) }\end{array}$ \\
\hline 0 & $32,44 a$ & $18,72 \mathrm{a}$ & $16,42 \mathrm{a}$ & $23,00 \mathrm{a}$ & $14,02 \mathrm{a}$ & $810,86 a$ & $2,93 a$ & $8,98 \mathrm{a}$ \\
\hline $750+2250$ & $25,11 \mathrm{~b}$ & $12,94 \mathrm{~b}$ & $13,74 \mathrm{~b}$ & $18,78 \mathrm{~b}$ & $11,59 \mathrm{~b}$ & $498,93 \mathrm{~b}$ & $1,41 \mathrm{ab}$ & $7,67 \mathrm{~b}$ \\
\hline $1000+3000$ & $23,22 \mathrm{~b}$ & $12,67 \mathrm{~b}$ & $12,86 \mathrm{~b}$ & $19,19 \mathrm{~b}$ & $11,28 \mathrm{~b}$ & $402,61 \mathrm{~b}$ & $1,22 \mathrm{~b}$ & $7,81 \mathrm{~b}$ \\
\hline $1500+4500$ & $23,39 \mathrm{~b}$ & $12,00 \mathrm{~b}$ & $13,45 \mathrm{~b}$ & $18,94 \mathrm{~b}$ & $11,17 \mathrm{~b}$ & $501,96 \mathrm{~b}$ & $1,42 \mathrm{ab}$ & $7,44 \mathrm{~b}$ \\
\hline
\end{tabular}

Médias seguidas de pelo menos uma mesma letra na coluna não diferem entre si, a 5\% de probabilidade, pelo teste de Tukey.

A aplicação do paclobutrazol teve efeito significativo na diminuição da altura da planta e da folhagem (Figuras 3 A e B), no comprimento de pedúnculo (Figura $3 \mathrm{C}$ ), no diâmetro de planta (Figura 3 D), na matéria seca do caule (Figura $3 \mathrm{E}$ ), na área foliar e na matéria seca das folhas (Figuras $3 \mathrm{~F}$ e G), proporcionando redução na altura das plantas em concentrações aproximadamente 100 vezes menores que as de clormequat e de daminozide. Conforme observado no presente experimento, TAYAMA e CARVER (1990) reportaram que a concentração de $15 \mathrm{mg}$ i.a./1 de paclobutrazol aplicada via pulverizações foliares proporcionou redução no diâmetro de plantas de gerânio cultivar 'Yours Truly'.

A concentração de $20 \mathrm{mg}$ i.a./1, de acordo com a recomendação de FONTENO (1992) e NELSON (2003), proporcionou altura de planta e de folhagem e diâmetro de planta (Figuras 3 A, B e D) adequados para plantas envasadas. As características número e diâmetro de inflorescências não foram afetadas por concentrações do retardante paclobutrazol.
Pelos resultados experimentais obtidos, concluiu-se que o uso de retardantes de crescimento não proporciona diferenças no tamanho do ciclo das plantas de gerânio. Pulverizações foliares com paclobutrazol por 3 vezes na concentração de $20 \mathrm{mg}$ i.a./1 resultaram em plantas com arquitetura desejada (Figura $4 \mathrm{D}$ ). O uso do paclobutrazol é recomendado quando existe conhecimento da cultura, das condições ambientais e experiência no uso de retardantes de crescimento, pois ele reduz o alongamento das hastes em concentrações de aproximadamente 1\% em relação aos retardantes de crescimento clormequat e daminozide.

Pulverizações foliares com clormequat por três vezes nas concentrações 1000 e $1500 \mathrm{mg}$ i.a./1 proporcionaram plantas com arquitetura de acordo com os parâmetros propostos (Figura 4 B). Por ser mais amplamente utilizado, seu uso é recomendado inclusive quando não existem vastos conhecimentos sobre o uso de retardantes de crescimento.

O daminozide promoveu redução no alongamento das hastes de plantas de gerânio, porém as altas concentrações necessárias para alcançar os resultados desejados podem 

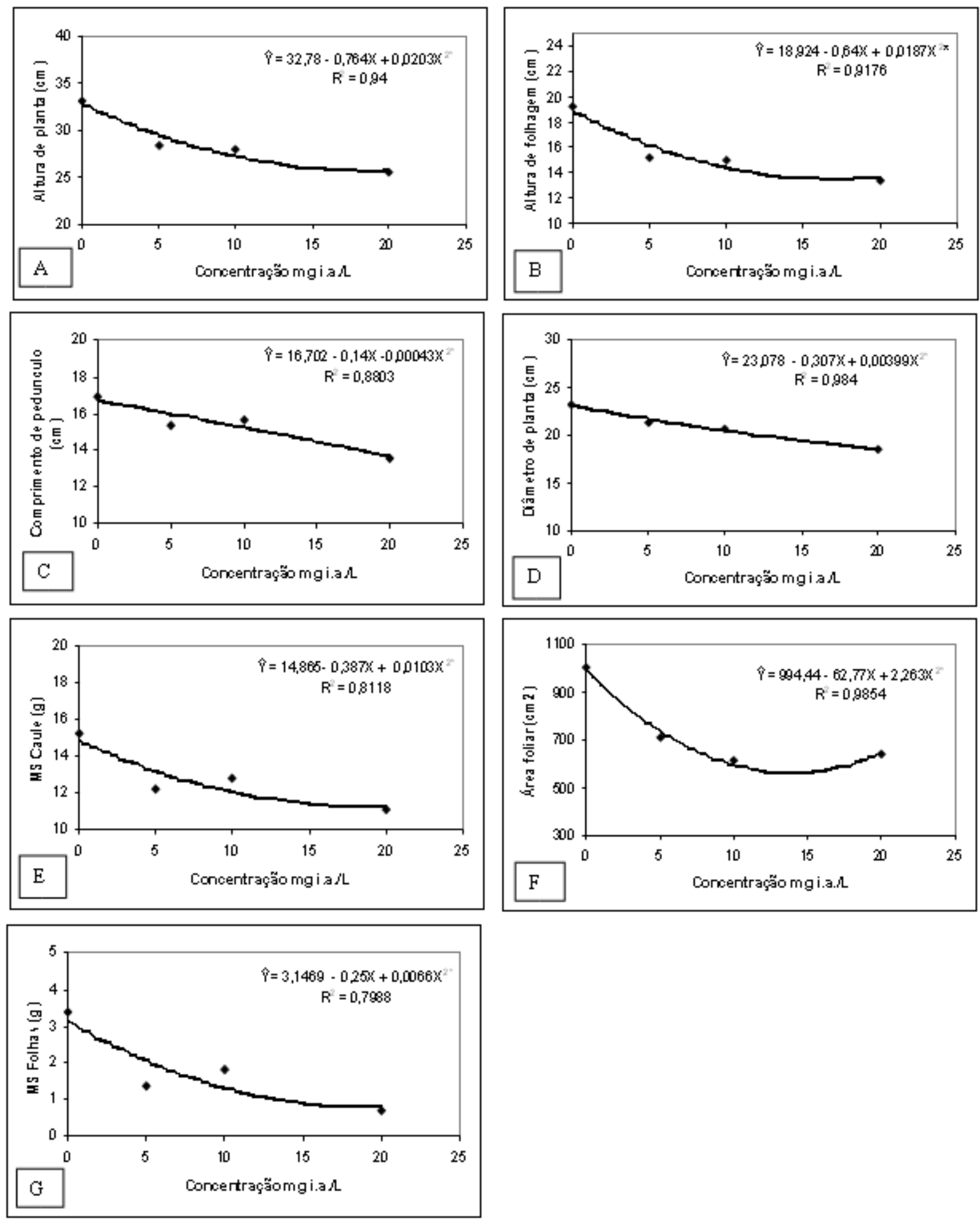

Figura 3. Efeito das concentrações (0, 5, 10 e $20 \mathrm{mg}$ i.a./l) de paclobutrazol aplicadas via pulverizações foliares para as características altura de planta (A), altura de folhagem (B), comprimento de pedúnculo (C), diâmetro de planta (D), matéria seca (MS) do caule (E), área foliar (F) e matéria seca (MS) das folhas (G) em plantas de gerânio das cultivares Grand Prix, Atlantis e Rocky Mountain, Viçosa, MG, 2004. significativo a $5 \%$ de probabilidade

Figure 3. Effect of foliar applications of paclobutrazol (concentrations - 0, 5, 10 e $20 \mathrm{mg} / \mathrm{l}$ ) on characteristics plant height (A), foliage height (B), stem lenght (C), plant diameter (D), shoot dry weight $(E)$, leaf area $(F)$ and leaf dry weight $(G)$ on geranium plants cultivars Grand Prix, Atlantis e Rocky Mountain, Viçosa, MG, 2004.

$$
\text { significant at } 5 \%
$$



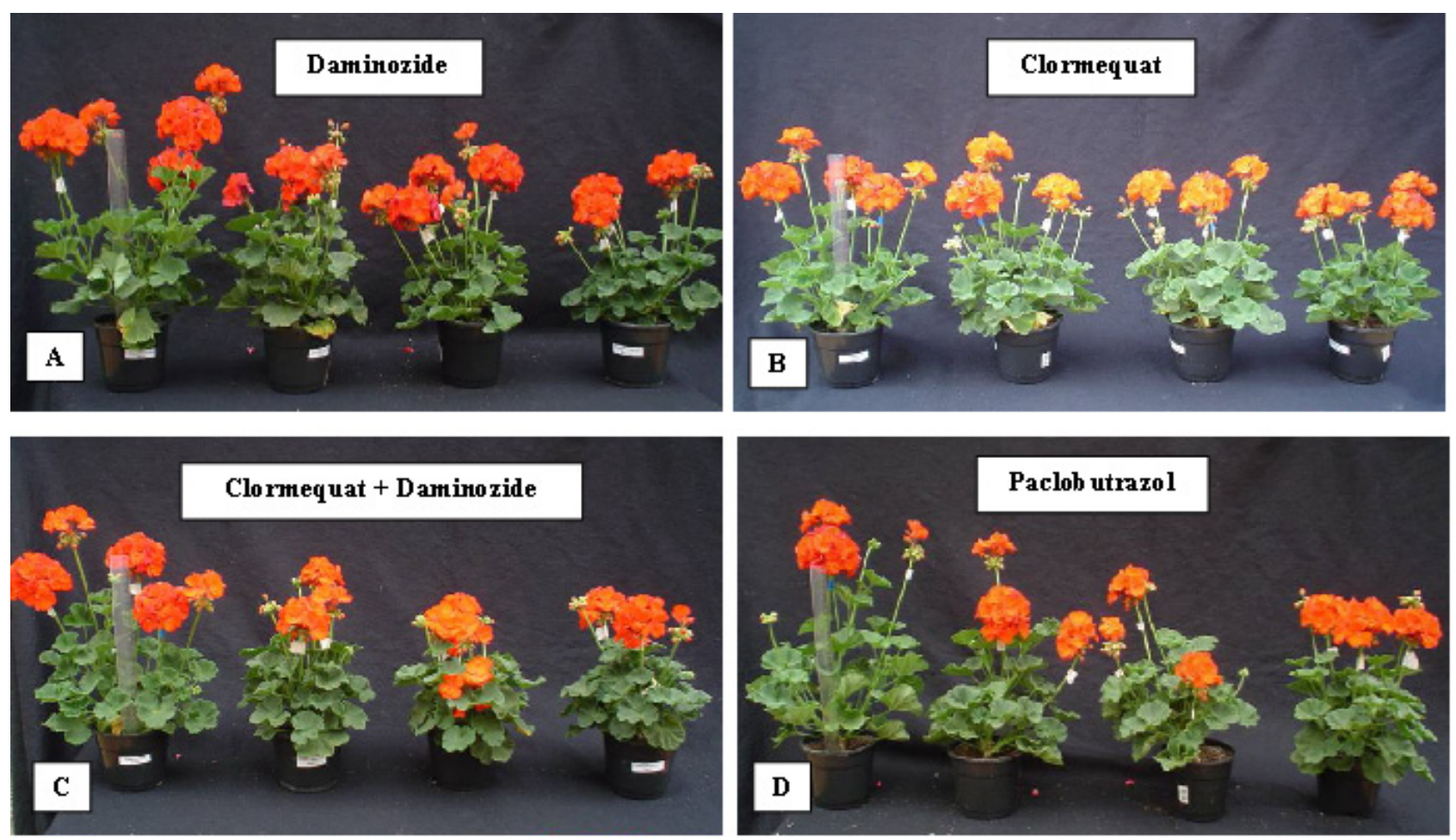

Figura 4. Plantas de gerânio cultivar Grand Prix submetidas às concentrações de 0, 1000, 2000 e 4000 mg i.a./1 de d(A); 0, 750, 1000 e $1500 \mathrm{mg}$ i.a./l de clormequat (B); 0, 750+2250, 1000+3000, 1500+4500 mg i.a./1 de clormequat+daminozide (C); 0, 5, 10 e $20 \mathrm{mg}$ i.a./1 de paclobutrazol (D). Em todas as figuras as concentrações estão representadas na seqüência da esquerda para a direita.

Figure 4. Geranium plants cultivar Grand Prix treated with concentrations of 0, 1000, 2000 e $4000 \mathrm{mg} / \mathrm{l}$ daminozide (A); 0, 750, 1000 e $1500 \mathrm{mg} / \mathrm{l}$ clormequat (B); 0, 750+ 2250, 1000+3000, 1500+4500 mg/l clormequat +daminozide (C); 0, 5,

10 e $20 \mathrm{mg} / \mathrm{l}$ paclobutrazol (D). In each picture, the 4 concentrations are represented on plants from left to right.

resultar em custos elevados, que implicariam a escolha de outro retardante de crescimento capaz de proporcionar melhor relação custo $x$ benefício (Figura 4 A).

As concentrações da mistura de retardantes utilizada proporcionaram maior intensidade de controle do crescimento das plantas quando comparada ao uso do clormequat e daminozide separadamente (Figura 4 C). Não foram observados sintomas de fitotoxidez, porém o uso da mistura implica manejo mais trabalhoso.

\section{REFERÊNCIAS}

BARRETT, J.E.; NELL T.A. Factors affecting efficacy of paclobutrazol and uniconazole on petunia and chrysanthemum. Acta Horticulturae, East Lansing v. 272, p. 229-234, 1990.

BARRETT, J.E.; HOLCOMB, E.J. Growth regulating chemicals. In: WHITE, J. Geraniums IV. (ed.) West Chicago: BallPublishing, 1993. cap. 7, p. 65-74.

CASTRO, C.E.F. Cadeia produtiva de flores e plantas ornamentais. Revista Brasileira de Horticultura Ornamental, Campinas, v.4, n.1/2, p.1-46, 1998.

CLARO, D.P.; SANTOS, A. C. e CLARO, P.B.O. Um diagnóstico do agregado da produção de flores do Brasil. Revista Brasileira de Horticultura Ornamental, Campinas, v.7, n.1, p.9-15, 2001.

CHRISTENSEN, O.V.; FRIIS, K. Research and development of unknown pot plants. Acta Horticulturae, n. 205, p. 33-37, 1987.

DAVIS, T.D.; CURRY, E.A. Chemical regulation of vegetative growth. Critical Reviews in Plant Sciences, Philadelphia v. 10, n. 2, p. 151-188, 1991.

DAVIS, T.D.; STEFFENS, G.L.; SANKHLA, N. Triazole plant growth regulators. Horticultural Reviews, Portland, v. 10, p. 63-105, 1988.

DICKS, J.W.; CHARLES-EDWARDS, D.A. A quantitative description of inhibition of stem growth in vegetative lateral shoots of Chrysanthemum morifolium by $N$-dimethylaminosuccinamic acid (Daminozide). Planta, Berlim, v. 112, p. 71-82, 1973.

FAUST, J.; LEWIS, K. Tank-Mixing PGRs. Greenhouse Product News, v. 13, n. 2, 2003. Disponível em: http:// www.gpnmag.com. Acesso em: 27/09/2005. 
FONTENO, W. C. Geraniums. In: LARSON, R. A. Introduction to Floriculture. $2^{\text {nd }}$ ed. San Diego: Academic Press, 1992. chap.18, p.451-475.

HAMZA, A.M.; KORANSKI, D.S.; ROGERS, M.N. The effects of sequential applications of different chemical growth regulators on growth and flowering of $F_{1}$ hybrid geraniums (Pelargonium $\mathrm{x}$ hortorum Bailey). Journal of the American Society for Horticultural Science, Alexandria v. 106, n. 3, p. 299-303, 1981.

HOLCOMB, E.J.; WHITE, J.W. A technique for soil application of a growth retardant. HortScience. Alexandria v. 5, n. 1, p. 16-17, 1970.

IBRAFLOR, Instituto Brasileiro de Floricultura. Relatório do Diagnóstico da Produção de Flores e Plantas Ornamentais Brasileira. Campinas: 2003.

JOSÉ, A.R.S. Reguladores vegetais em flores de vaso. Disponível em: http://www.uesb.br/flower/regulador.html. Acesso em: 16/05/2005.

LEWIS, K.P; FAUST, J.; SPARKMAN IV, J.D. B-Nine + Cycocel: the advantages for poinsettias and pansies. Greenhouse Product News, Arlington Heights v. 12, n. 7, 2002. Disponível em: http://www.gpnmag.com. Acesso em: 25/10/2005.

MEJIAS, R. .; RUANO, M. C. El cultivo industrial de plantas em maceta. Ediciones de Horticultura, Barcelona, 1990. 664p.
NELSON, P. Chemical growth regulation. In: NELSON P. V. Greenhouse operation and management. $6^{\mathrm{a}}$. ed. Upper Saddle River, N.J.: Prentice Hall, 2003. cap. 13, p. 433- 462.

OLSEN, W.W.; ANDERSEN, A.S. Growth retardation of Osteospermum ecklonis. Acta Horticulturae, Kyoto v. 397, p. 129-137, 1995.

RADEMACHER, W. Growth retardants: Effects on gibberellin biosynthesis and other metabolic pathways. Annual Reviews in Plant Physiology and Plant Molecular Biology, Minneapolis v.51, p. 501-531, 2000.

SAS Institute Inc. SAS/STAT ${ }^{9}$ 9.1 User's guide. Cary, N.C.: 2004.

SACHS, R.M.; HACKETT, W.P. Chemical inhibition of plant height. HortScience, Alexandria, v. 7, n. 5, p. 440447, 1972.

SEMENIUK, P.; TAYLOR, R. Effects of growth retardants on growth of geranium seedlings and flowering. HortScience, Alexandria, v. 5, n.5, p. 393-394, 1970.

TAYAMA, H.K.; CARVER, S.A. Zonal geranium growth and flowering responses to six growth regulators. HortScience, Alexandria, v.25, n. 1, p.82-83, 1990.

TAYAMA, H.K.; CARVER, S.A. Residual efficacy of uniconazole and daminozide on potted 'Bright Golden Anne' chrysanthemum. HortScience, Alexandria, v.27, n. 2, p. 124-125, 1992. 